\title{
Hardware/Software Obfuscation against Timing Side-channel Attack on a GPU
}

\author{
Elmira Karimi, Yunsi Fei, David Kaeli \\ ECE Department, Northeastern University Boston, MA 02115 USA \\ \{elmirakarimi, yfei, kaeli\}@ece.neu.edu
}

\begin{abstract}
GPUs are increasingly being used in security applications, especially for accelerating encryption/decryption. While GPUs are an attractive platform in terms of performance, the security of these devices raises a number of concerns. One vulnerability is the data-dependent timing information, which can be exploited by adversary to recover the encryption key.

Memory system features are frequently exploited since they create detectable timing variations. In this paper, our attack model is a coalescing attack, which leverages a critical GPU microarchitectural feature - the coalescing unit. As multiple concurrent GPU memory requests can refer to the same cache block, the coalescing unit collapses them into a single memory transaction. The access time of an encryption kernel is dependent on the number of transactions. Correlation between a guessed key value and the associated timing samples can be exploited to recover the secret key.

In this paper, a series of hardware/software countermeasures are proposed to obfuscate the memory timing side channel, making the GPU more resilient without impacting performance. Our hardware-based approach attempts to randomize the width of the coalescing unit to lower the signal-to-noise ratio. We present a hierarchical Miss Status Holding Register (MSHR) design that can merge transactions across different warps. This feature boosts performance, while, at the same time, secures the execution. We also present a software-based approach to permute the organization of critical data structures, significantly changing the coalescing behavior and introducing a high degree of randomness. Equipped with our new protections, the effort to launch a successful attack is increased up to $1433 X \times 178 X$, while also improving encryption/decryption performance up to $7 \%$.
\end{abstract}

\section{INTRODUCTION}

Graphic Processing Units (GPUs) have become the accelerator of choice in a number of compute-intensive applications. GPUs achieve high throughput by leveraging thread-level parallelism of applications on thousands of simple cores. The range of applications that can leverage GPU acceleration spans deep learning models, computational physics, and security functions such as data encryption and signing [1], [2], [3], [4], [5].

CPUs are now offloading encryption workloads to GPUs, given their efficiency. Encryption/decryption operations are commonly present in security libraries and they can benefit greatly from the high performance provided by a GPU [6], [7], [8], [9], [10], [11]. Iwai et al. [7], Li et al. [12] and Schonberger [13] each developed GPU implementations of the Advanced Encryption Standard (AES) algorithm, demonstrating double-digit speedups on a GPU compared to a CPU. However research has shown that GPUs are vulnerable to attacks that leverage the physical properties associated with applications execution on the device, a class of vulnerabilities called side-channel attacks [14], [15], [16], [17]. Although some companies have developed secure encryption cores, these designs are specifically implement a single algorithm (e.g., AES) [18]. Side-channel attack methods can be used to attack different table-based algorithms. Therefore we need a more comprehensive defense strategy. Timing side-channel attacks have typically targeted the memory hierarchy of a GPU. If the attacker can measure the execution time of an encryption, and with knowledge of the encryption algorithm's layout in memory, the encryption keys can be recovered through correlation analysis [15], [16], [17]. RCoal [19], has been proposed as a countermeasure that can change the warp size and thread allocation randomly. Unfortunately, this solution hurts performance significantly due to reduced memory hierarchy efficiency, while only slightly improving security.

In this paper, we introduce a series of hardware and software countermeasures against GPU coalescing attacks. Given the fact that attackers can use the relationship between execution time and number of memory accesses to launch an attack, our approach attempts to decorrelate the measured timing signal and memory access pattern. In most GPU memory systems, the coalescing unit can dynamically merge multiple memory requests across different threads into a single memory access, called a transaction. Given the organization of the data structures used during encryption, and their associated key-dependent memory access behavior, the memory access pattern produces a timing channel [17].

To confuse the attacker, we first enhance the hardware by randomizing the width of the coalescing unit to reduce the Signal-to-Noise Ratio (SNR) of the timing sample. By modifying the width of this unit during program execution, we can introduce randomness in terms of the number of memory transactions generated. In order to further reduce the SNR, we utilize multiple coalescing unit widths across different cache lines. By dynamically changing the width of coalescing unit for different cache lines, we can introduce another dimension to the noise injected. Since each Streaming Multiprocessor (SM) in a NVIDIA GPU has its own coalescing unit, we use a combination of different transaction sizes for each SM to introduce additional noise to the timing channel. We also redesign the Miss Status Holding Registers (MSHRs) to provide miss tracking across SMs, which not only adds some additional noise to the timing channel, but also improves performance. 
In order to explore these changes in hardware, we utilize an execution-driven GPU simulator.

For our software approach, we also focus on the memory model of the GPU. During AES encryption/decryption we need to access several look-up tables stored in GPU memory. We introduce a software-based lookup table hashing algorithm to obfuscate the associated access pattern to one of these tables. This helps to hide the number of transactions observed by the attacker through the measured timing sample.

The rest of this paper is organized as follows. Section 2 covers background on GPU microarchitecture and describes a GPU-based coalescing attack. Section 3 provides an introduction to our methodology. In Section 4, we characterize the timing channel present on a GPU, as well as the design of our hardware/software countermeasures based on changing the coalescing unit width, modifying the MSHR design and applying look-up table rotation. Then we evaluate how well we can thwart a timing attack in Section 5. Finally, in Section 6 , we conclude the paper.

\section{BACKGROUND AND RELATED WORK}

\section{A. GPU Architecture}

In contrast to CPUs, GPUs typically provide many cores. NVIDIA refers to these cores as Streaming Processors (SMs), while AMD calls them Compute Units (CUs). In this paper we will use NVIDIA terminology and provide a brief review of the Fermi GPU architecture. While Fermi is an older GPU architecture, it is used in our simulation infrastructure (GPGPU-Sim [20]), and most of the features discussed in this paper are present in later generations of NVIDIA GPUs, as well as AMD GPUs. So the attack strategies and defences proposed are relevant for a broad class of architectures. In this paper we use GPGPU-Sim based on the Fermi GTX480 GPU. architecture, which has 15 SMs. Each SM includes 32 CUDA cores, a load/store(LD/ST) unit, 4 Special Function Units (SFUs), a 64KB block of high speed on-chip memory (L1+Shared Memory) and an interface to the L2 dcache. The L2 cache is a unified shared cache across all the SMs. Each SM can run two warps, with each warp running 32 threads in lockstep. Warps are scheduled by a warp scheduler. Detailed configuration information is provided in Table I.

TABLE I

THE FERMi GTX480 ARCHITECTURE.

\begin{tabular}{|l|l|}
\hline Number of SMs & 15 \\
\hline $\begin{array}{l}\text { Number of Threads per } \\
\text { Warp }\end{array}$ & 32 \\
\hline L1 Data Cache Size & $48 \mathrm{~KB}$ \\
\hline L2 Cache Size & $768 \mathrm{~KB}$ \\
\hline Transaction Size & $64 \mathrm{~B}$ \\
\hline Memory Bus Width & $258 \mathrm{~B}$ \\
\hline MSHR Entries & 32 \\
\hline
\end{tabular}

Memory Coalescing: Threads within a warp run in a Single Instruction Multiple Thread (SIMT) fashion. As a result, each memory instruction in a warp will produce 32 memory requests, which are sent to the LD/ST unit. Each LD/ST unit includes a coalescing unit that inspects the addresses generated across the warp and collapses them into a minimum set of memory transactions. The size of each transaction is equal to one cache line. Accesses are non-blocking, such that memory can handle multiple requests concurrently. Wang et. al [21] proposed an intra-cluster coalescing unit to merge memory requests from different SMs in a cluster.

Miss Status Holding Register Functionality: Every SM has a private L1 cache. On an L1 read miss, an entry in the Miss Status Holding Registers (MSHRs) will be allocated for tracking the miss. Each outstanding request to the L2 cache is tracked by an MSHR entry. Once the L2 cache responds, the MSHR entry will be released. Each MSHR entry can be shared by multiple accesses in an SM in order to avoid redundant entries, though redundant entries from across different warps may cause a performance loss. Tuck et. al [22] described sharing MSHR entries across L1 banks, improving bandwidth management to L2.

\section{B. Side-channel Attacks}

Side-channel attacks exploit information recorded during execution of encryption/decryption. There are many types of attacks and countermeasures in different levels of the design [23], [24], [25], [26], [27], [28]. The memory hierarchy is one of the most common targets for the attacker. There are two main types of memory attacks: access-driven and timedriven attacks. In an access-driven attack, the attacker exploits the principle of cache sharing to observe the victim's memory access behaviour. The adversary intentionally creates contention on the shared cache resource to be able to infer if certain addresses have been accessed by the victim. Alternatively, a time-driven attack is based on measuring the execution time of an encryption/decryption and establishing a correlation between this timing value and an embedded secret.

Most previous studies of side-channel attacks, as well as attack countermeasures, have targeted CPU devices. Bernstein [29] proposed a simple class of timing attack strategies that leverage the layout of data structures in the memory hierarchy of a CPU and used the correlation between the data structure index and the measured time to recover the encryption key. Tromer et al. [30] describe several accessdriven attacks and countermeasures against such timing. These methods attempt to obscure the timing channel by introducing time constant algorithms, masking the access pattern by disabling shared memory or performing random permutations and preloading. Both software and hardware approaches have been used, but the main problem with most of these obfuscation techniques is that they come with significant performance costs, rendering them infeasible or costly to implement.

Given the growing popularity of GPU platforms, it is important to thoroughly evaluate vulnerabilities of these highperformance devices, as well as employ effective countermeasures against various attacks. The most important difference between CPUs and GPUs is the source of the execution time variation. In CPUs, the variations in timing are the result of cache misses (or hits), providing a clean timing channel. 
While on a GPU, instead of tracking cache hits and misses, timing variations are a result of memory request management by the GPU's coalescing unit. While the degree of threadlevel concurrency is very high on a GPU, the noise associated with this concurrency is not enough to obscure the timing channel [15], [16], [17]. The attacker generally only observes a single timing value associated with the execution of all the threads in a kernel. Although, the correlation between the execution time and the memory access pattern becomes weaker as the degree of concurrency is increased, the GPU memory reference pattern is mainly a function of the plaintext input and encryption/decryption algorithm. An attacker can track data access patterns using timing. Even if the programmer chooses to store the encryption data structures in shared memory, a partition of memory only accessible to each Streaming Processor on the device, a successful attack is still possible. The good news is that the same memory features that provide attackers with an attractive attack surface, can also be used as a form of protection.

\section{Baseline Attack}

Memory coalescing is a common feature that has been used by GPU designers to improve memory throughput. This mechanism also provides a strong timing channel, since the effectiveness of the coalescing unit is a function of the memory address pattern generated across threads. An attacker can use this timing information to quantify the number of transactions, and eventually derive the encryption key by computing the correlation between the measured time and number of transactions. This attack scenario was first identified by Jiang et al. [16], where the attacker remotely sends a large number of random plaintext inputs to the victim. The victim uses a GPU to encrypt the data and the attacker finally collects the resulting ciphertext and the execution time. The attack is repeated many times (typically $100 \mathrm{~K}$ to $1 \mathrm{M}$ samples are collected).

$$
C_{j}=T_{4}\left[t_{i}\right] \oplus k_{j}: 0<j<15,0<i<15
$$

This attack method is adopted as our baseline methodology. Based on this scheme, we will introduce randomness during encryption to obfuscate the timing channel. Jiang et al. described a T-table implementation of AES encryption (ECB mode). We implement the same scheme in CUDA, following the OpenSSL 0.9.7 library implementation. The CUDA kernel takes 16 bytes of plaintext and 16 key bytes, and outputs ciphertext. The key bytes are expanded to 160 bytes, because in total AES has 10 rounds. First, the 16-byte plaintext is XORed with the first round key. Then during the next 9 rounds, a combination of the four T-table lookups $\left(T_{0}, T_{1}, T_{2}\right.$ and $\left.T_{3}\right)$ are XORed with the key. For the last round, another T-table, $T_{4}$, are performed. As there is no MixColumn operation, the ciphertext can be derived using Equation 1.

In our CUDA implementation, the entire encryption is implemented in a kernel. Each thread in the kernel takes 16 bytes of plaintext, performs an independent encryption, and

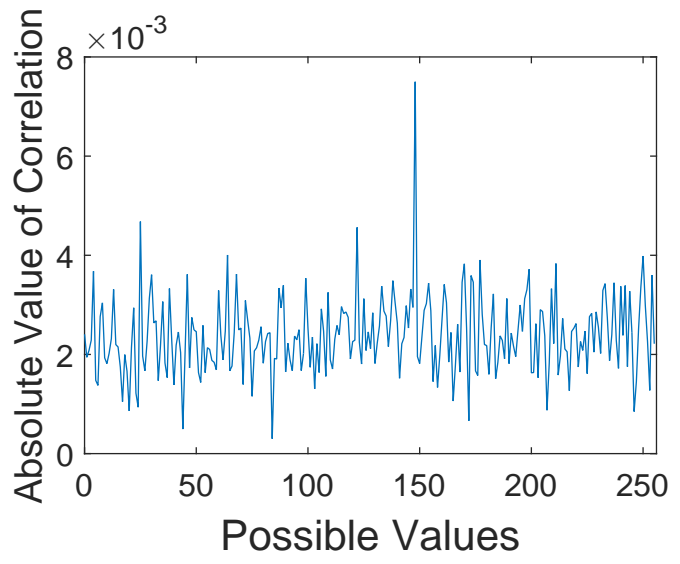

Fig. 1. The absolute value of the correlation for possible values of $k_{5}$.

produces 16 bytes of ciphertext. A warp contains 32 threads, so 32 encryptions are performed together. Each T-Table has 256 4-byte elements (1 KB in total). The T-table is stored in global memory.

In the attack scenario, each key byte is guessed (from the values 0 to 255). By knowing the ciphertext of each sample and using Equation 2, the attacker can produce all indices $\left(t_{i}\right)$. Equipped with a coalescing unit, the 32 concurrent memory requests result in a smaller (and computable) number of memory transactions. The attacker only needs to know the width of the coalescing unit. For example, if the transaction size is 32 bytes, each memory transaction would contain 8 consecutive addresses. To recover the transaction index, the attacker simply shifts the original index by $\log _{2} 8$ (i.e., 3). After determining the number of transactions based on the cipherthext, the attacker can calculate the correlation between the measured execution time and the predicted number of transactions under a key byte guess.

For a key byte, there are 256 possible values. The attacker can calculate the set of 256 correlation values using the execution time and the number of transactions for each key guess. The value with the highest correlation between the execution time and the number of transactions will be associated with the correct key guess. The attacker will repeat this process for the other 15 bytes of the key and find the highest correlation for each. Figure 1 shows the correlation of all possible values of $k_{5}$, using 500,000 timing samples.

$$
\begin{gathered}
t_{i}=T_{4}^{-1}\left[C_{j} \oplus k_{j}\right]: 0<j<15,0<i<15 \\
\text { III. Our Methodology }
\end{gathered}
$$

Typically, the degree of difficulty associated with an attack can be measured by the number of timing samples needed to recover the key. An ideal countermeasure increases the number of samples required for the attack, without hurting execution performance. Kadam et al. [19] changed the number of threads that are grouped together in a warp randomly. While introducing randomness to help protect a device, GPUs are 
designed to execute regular (e.g., dense matrix) operations, where the execution time is well behaved. So reducing the warp size in order to introduce noise in the timing channel should greatly impact performance.

We introduce a series of hardware/software countermeasure based on commonly design principles used in most GPU memory systems. Given the fact that the attacker can use the relationship between time and the associated memory access pattern, our approach attempts to reduce the correlation between number of memory accesses and the measured timing signal.

\section{A. Obfuscation in Hardware}

1) Randomizing The Width of the Coalescing Unit: The transaction size of coalescing unit is equal to the size of an L1 cache line. The address range of the coalescing unit is always fixed. Across the threads in a warp, each address pattern will result in a predictable number of cache line accesses. In order to confuse any attacker who is tracking memory behavior, we propose an obfuscation approach that dynamically modifies the coalescing unit. By changing the range of addresses that result in a transaction, we render the execution time associated with a memory address pattern as non-deterministic. For example, if the cache line size is $l$ bytes, the basic coalescing unit maps addresses from the range 0 to $l-1$ into the first cache line, but with our coalescing unit, the width of coalescing unit is randomly selected to be a fraction of the total cache line size. This will introduce a source of noise, and by randomly changing the number of transactions, this will significantly increase the number of executions required to launch a successful coalescing attack.

We start by randomizing the width of the coalescing unit based on a normal probability density function. In this step, the width of the coalescing unit is randomly chosen, but the value is fixed for each individual cache line. To inject more noise, we can use different coalescing widths for different cache lines. One simple way to do this is to generate a random width for every cache line. Given that an L1 cache has a large number of cache lines, generating a random value for the width to be used for every line would be costly. In order to reduce the overhead, we generate a limited number of random widths, for example 16 , and use this set of widths for every 16 cache lines, and then repeat the pattern through the whole cache. This approach introduces additional noise into the timing channel, and makes it significantly harder for an attacker to recover the key.

2) A Hierarchical MSHRs for Increased Obfuscation Strength: MSHRs help to manage memory bandwidth when encountering L1 misses. GPUs provide a private set of MSHRs for each SM. Kernels tend to fully utilize all SMs in order to exploit the highest degree of parallelism available on a GPU. As a result, there can be a varying number number of misses experiences across different SMs to the unified L2, which results in a performance loss due to L2's limited bandwidth and the number of MSHRs per SM available. To provide further obfuscation, while also considering performance, we present a hierarchical MSHRs design, where at the first level, each SM has its own set of MSHRs. Then at the second level, the
MSHR entries are shared across all SMs. This can benefit both the security and performance of the system. In terms of security, a hierarchical MSHRs will reduce the number of L1 misses from different SMs and hide a portion of the latency, which introduces additional noise into the timing channel. Our proposed design should also improve memory performance as compared to current GPU-based MSHRs.

\section{B. Obfuscation in Software}

Changing the T-table mapping in the cache is a commonly proposed countermeasure which attempts to randomly permute the address mapping using either software or hardware. Wang et al. [31] proposed novel cache design named RPcache, which can only defeat access-driven attacks. Brickell et al. [32] considered obfuscation that employed a software solution, though a fixed permutation only obfuscates the timing channel

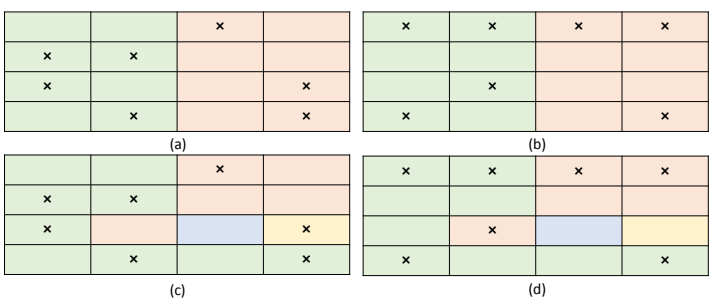

Fig. 2. Hardware and Software Obfuscation using: a) a fixed coalescing unit width, b) a fixed coalescing unit width with rotated accesses, c) a dynamic coalescing unit width (for row 1 to 4 the widths are 2, 2, 1 and 4, respectively), and (d) a dynamic coalescing unit width combined with rotated accesses.

temporarily until the attacker figures out the permutation algorithm. In this paper we propose a GPU-oriented random rotating algorithm that works by rotating T-table addresses, changing the permutation over time. The frequency of applying rotation can be controlled to minimize the overhead, while providing the right level of obfuscation. Dynamic rotation also can exploit the parallelism of a GPU to prevent performance loss.

Our approach effectively disassociates elements stored in a cache line and re-associates them with new elements in other cache lines. As an example, suppose that we have $n$ elements in the T-table that will be stored in $l$ continuous cache lines and each cache line contains $m$ elements. Figure 2(a) shows a simple case in which the look-up table has 16 elements and each cache line can store 4 elements. As we can see, this is a two-dimensional structure, where the rows represents cache lines. In this example, we have 7 random accesses, where the number of accessed cache lines (i.e., rows) is 4 . The attacker can use this number to launch an attack by finding the correlation between the execution time and the number of cache accesses. One approach to obfuscate the number of rows accessed is to change the mapping of each entry, column-by-column, as shown in Figure 2(b), where columns 1, 2, 3 and 4 are shifted by $2,3,0$ and 1 , respectively This rotation changes the access pattern as shown, resulting in changes to the row accessed from 4 to 3 .

Figure 2 also shows the impact of obfuscation by changing the coalescing unit width, combined with rotation in the T-table. 
In Figure 2(a), we show results using a fixed coalescing unit width $(=2)$ with different colors. In Figure 2(b), we combine a fixed coalescing unit width with the T-table rotation (as described before). Figure 2(c) shows results for dynamically changing the width of the coalescing unit for different rows. Figure 2(d) shows results when dynamically changing the coalescing unit width, combined with T-table rotation. The number of rows accessed is $6,5,5$ and 4 for cases a-d, respectively.

\section{HARDWARE/SOFTware COUNTERMEASURES}

In this section, first we present a series of hardware countermeasures based on a redesign of the GPU's coalescing unit. Then we provide details of our hierarchical MSHR design. Our solution improves performance and provides further protection. Then we discuss our software approach, which randomly rotates columns of the T-table in the GPU's global memory.

\section{A. Randomizing the Width of the Coalescing Unit}

As discussed earlier, we use GPGPUsim to evaluate a timing attack of an AES running on a Fermi GPU. In this simulator, we set the memory transaction size to 64 bytes, which is equal to L1 cache line size. To study the effects of changing the width of the coalescing unit on the execution time of a kernel, we first develop a simple kernel to learn the behavior of the coalescing unit and then design the countermeasure based on our observation.

1) benchmarking Observations: We developed a microbenchmark kernel to study the impact of changing the width of the coalescing unit. The kernel loads an array of floats from global memory, where each warp generates 32 loads. We set the array indices to access $n$ unique addresses. We modify this pattern, varying the value of $n$ from 1 to 32 over each kernel, for 32 threads. We vary the width of the coalescing unit, setting this value to $8,16,32$ and 64 bytes. Our design space includes $32 \times 4$ configurations. Each microbenchmark kernel is run 10,000 times per configuration. We collect the corresponding timing samples for each configuration, $128 \times 10^{4}$ timing samples in total. Figure 3 presents the measured execution time, versus the number of unique addresses, for coalescing unit widths of 32 and 64 bytes. We added a random noise to the simulator's timing to simulate the real hardware. As the results show, the execution time is linearly proportional to the number of unique addresses, and changing the width of coalescing unit changes the slope of this line. Using regression analysis, we can construct a linear equation that expresses the relationship between the time samples and the number of unique addresses. The coefficients of this equation are found using a least-squares approximation method, which minimizes the distance between the actual timings and the expected ones. In equation $3, t_{e}, n$ and $\epsilon$ are the execution time, the number of unique accesses and the error due to noise, respectively. We use regression analysis to find $\beta_{1}$, which represents the slope of the line and $\beta_{0}$ that is a constant.

$$
t_{e}=\beta_{1} n+\beta_{0}+\epsilon
$$

The attacker can use the fact that the time measured is linearly proportional to the number of unique accesses, and can be inferred from the execution time. But when varying the width of the coalescing unit, we observe a less tractable relationship between time and the memory addresses. This confirms that the design of the coalescing unit can impact the leakiness of the GPU. One metric that can be used to measure the leakiness of an architecture is SNR. A higher SNR is desirable for the attacker. Therefore, to protect a GPU from a side-channel attack, we should try to reduce the SNR. The SNR for the linear model produced by our regression analysis (Equation 3) is calculated by Equation 4, where $\sigma_{s}^{2}$ and $\sigma_{\epsilon}^{2}$ represent the variance of the signal and the noise.

$$
S N R=\frac{\sigma_{s}^{2}}{\sigma_{\epsilon}^{2}}=\frac{\beta_{1}^{2} \sigma_{n}^{2}}{\sigma_{\epsilon}^{2}}
$$

If the access pattern and noise remain constant, increasing the slope of the line results in a higher SNR. In other words, $\beta_{1}$ captures how likely the attacker can distinguish addresses $i$ and $i+1$ when she records the corresponding execution times $\left(t_{e}(i)\right.$ and $\left.t_{e}(i+1)\right)$. The first four rows of Table II show the characteristics of the linear model while varying the width of the coalescing units. As we can see, by increasing the width of the coalescing units, $\beta_{1}$ is reduced, which results in a lower SNR based on Equation 4. To calculate the SNR using Equation $4, \sigma_{\epsilon}^{2}$ is calculated in Equation 5.

$$
\sigma_{\epsilon}^{2}=\frac{\sum_{i=1}^{m}\left(t_{e}-\left(\beta_{1} n+\beta_{0}\right)\right)^{2}}{m}
$$

TABLE II

REGRESSION ANALYSIS CHARACTERIZATION.

\begin{tabular}{lccc}
\hline width of coalescing unit & $\beta_{1}$ & $\beta_{0}$ & SNR \\
\hline 8 & 19.463 & 346.2 & 1.8656 \\
\hline 16 & 11.42 & 344.3 & 0.9429 \\
\hline 32 & 6.032 & 343.6 & 0.4494 \\
\hline 64 & 3.601 & 336.9 & 0.1940 \\
\hline Fixed Random Width & 4.426 & 337.3 & 0.0985 \\
\hline Dynamic Random Width & 4.087 & 336.6 & 0.0050 \\
\hline
\end{tabular}
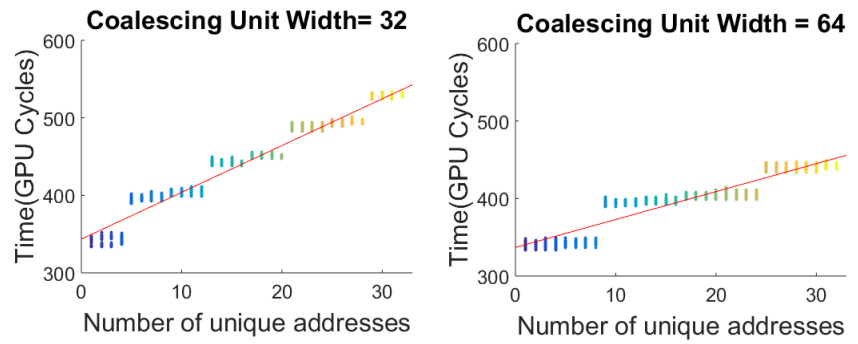

Fig. 3. Execution time of accessing 1 to 32 unique address with different width of coalescing unit

The results of our micro-benchmarking exercise illustrate how changes in the width of coalescing unit affect the SNR. 
Using the maximum width of the coalescing unit, the SNR is still high due to the lack of noise. One parameter to represent the noise is the standard deviation (STD) of the measured execution time. A lower STD means that the timing samples are less noisy. In our observations, when running our microbenchmark kernel 10,000 times, the STD is very low, meaning that the execution time is still highly correlated with the number of unique accesses. So the attacker can filter out noise by running a reasonable number of samples. To introduce more noise into the system, we randomly change the width of the coalescing unit to increase $\sigma_{\epsilon}^{2}$, and eventually, reduce the SNR. This randomness can be fixed and dynamic, which will be described next.

2) Using a Fixed Random Width for the Coalescing Unit: Introducing fixed randomness means that during each kernel run, the width of the coalescing unit is randomly chosen, but the width is fixed for all cache lines. Here, we have used 4 different coalescing unit width values as our reference set. The width of coalescing unit is $2^{k}$ bytes, where $k$ is varied from 3 to 6 . The maximum width of the coalescing unit (64 bytes) is equal to the width of an L1 cache line. The total number of timing traces collected is still 10,000 and the probability density function (PDF) representing the coalescing unit width is a skewed normal distribution, with a mean $k=5$, as shown in Figure 4. By using this distribution, the probability of choosing a larger transaction size is much higher (e.g., for only 500 out of 10,000 runs, we see 8 -byte transactions). If we run 10,000 samples while selecting a random width for the coalescing unit, the SNR is reduced (as shown in Table II) due to the increased noise.

3) Using a Dynamic Random Width for the Coalescing Unit:: As we observed, randomizing the width of coalescing unit increases the amount of noise and reduces the SNR. In regression analysis, the variance of the noise is equivalent to the sum of the squared distances between the predicted and actual values. Randomly changing the width of the coalescing unit increases this distance, resulting in more noise.

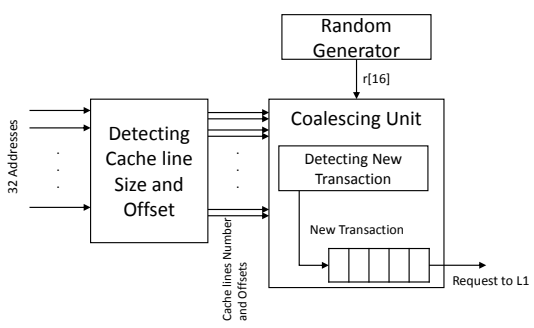

Fig. 5. Redesign Coalescing Unit for Dynamic Random Width

But we still utilize a fixed random value selected from a reference set. Another level of randomness would be to use different widths for different cache lines. But generally speaking, supporting a unique random width for every cache line has a huge overhead. Therefore, we choose a limited number of cache lines and produce $n$ different width values and repeat the random width pattern for every $n$ cache lines. Here, we choose 16 cache lines due to the T-table size, which occupies 16 cache lines. A high-level design for this functionality is shown in Figure 5.

In this design, a pseudo-random number generator generates 16 values during each kernel run, $r[16]$, from the set $1,2,4,8$, recognizing that each cache line width should be split into $r[i]$ parts that we call subtransactions. Once a request is issued by a warp, the cache lines and offsets within the cache lines are calculated for all 32 requests. At this point, a traditional coalescing unit would check each request to see if there is a memory request being processed for this cache line being issued by another thread. If a match is found, the current request will be coalesced and checking will move to the next request.

In our proposed design, when the coalescing unit receives a request and finds an access referencing the same cache block in the ordered memory request queue, it will first check the number of subtransactions within the cache line based on the $r$ array entry, indexed as follows: $r$ [cachelinenumber\%16]. Then the width of each subtransaction will be calculated as: 64 . We refer to this width as the subtransacrion width. The coalescing unit will resolve the subtransaction
tion widis number of the current request (0 to $r$ [cachelinenumber\%16]1) based on the request's offset, and if there is a queued request with the same cache line and subtransaction number for the same cache line, the current request can be coalesced. Otherwise, a new transaction will be sent to L1. While this new transaction is possibly unnecessary given that there is already an outstanding transaction for the same cache line underway, the added time will help obfuscate this timing channel. We provide the SNR values of this case in Table II. Using this method makes the design more resilient to the attack due to randomly chaning the pattern in every single kernel run.

\section{B. Hierarchical MSHR Design}

As we discussed earlier, each SM in our architecture has a set of 32 MSHRs for tracking L1 misses. One issue with this microarchitecture is that threads across different SMs could request the same data from $\mathrm{L} 2$, generating redundant requests and producing a bottleneck at L2. This issue can significantly impact performance when many SMs are making requests to L2. In order to avoid this issue, we introduce a second level of MSHRs. This set of MSHRs is unified and shared across all SMs, with each MSHR having 32 entries. In this design, each L1 miss first allocates an entry in the MSHR local to the SM issuing the request to L2. These requests arrive at the second-level MSHRs, and if there are no outstanding requests issued by other SMs for the same L2 cache line in the unified MSHRs, the request will be sent to the L2 cache. This design will increase performance whenever the competition for L2 access by multiple SM is high. Although a unified MSHR 
set is a shared hardware structure across SMs, it cannot be an attack surface. First of all, in most GPU applications, the GPU resources are fully utilized such that the attacker would not be able to run their application concurrently with victim's. Second, a kernel would be run on several SMs and all of these SMs would send L2 requests via the MSHRs. Therefore, even though the attacker can get an SM to observe the requests of a unified MSHR, it is very challenging to track the requests coming from different SMs that are executing concurrently.

While our MSHR design has the potential of improving performance, the second-level unified MSHRs will introduce another source of obfuscation. A hierarchical MSHR set can merge and collapse different incoming requests within a range of 128 bytes(L2 cache line size). This architecture will lower the SNR due to hiding the latency of L1 misses. In Equation 3 we have modeled the time as a linear function of the number of transactions. Suppose that we have $\beta_{10} \mathrm{~L} 1$ misses resulting from $\beta_{1}$ memory accesses. We can rewrite Equation 3 as Equation 6, where $h$ is the hit time and $m$ is the miss time for the $\mathrm{L} 1$ cache and $m=m_{0} h$.

$$
t_{e}=\left(\beta_{10} m_{0}+\left(\beta_{1}-\beta_{10}\right)\right) h n+\beta_{0}+\epsilon
$$

When we use a hierarchical MSHR set, there is some possibility that we will reduce the miss penalty for some L2 requests. This nondeterministic behavior will add noise to $m_{0} h$ by reducing the miss time to $m_{\text {noise }} h$, which is in the range of the time required to service the outstanding miss.

If the requests can be merged with probability $p_{m_{0}}$, then Equation 6 can be rewritten as Equation 7. In Equation 8, $\epsilon_{0}$ is the noise generated using our hierarchical MSHR set.

$t_{e}=\left(\beta_{10} m_{0}+\left(\beta_{1}-\beta_{10}\right)\right) h n+\beta_{0}+\epsilon-p_{m_{0}}\left(m_{0}-m_{n o i s e}\right) \beta_{10} n h$

$$
t_{e}=\left(\beta_{10} m_{0}+\left(\beta_{1}-\beta_{10}\right)\right) h n+\beta_{0}+\epsilon+\epsilon_{0}
$$

With this architecture the SNR will be lowered due to adding $\epsilon_{0}$ as:

$$
S N R=\frac{\beta_{1}^{2} \sigma_{n}^{2}}{\sigma_{\epsilon}^{2}+\sigma_{\epsilon_{0}}^{2}}
$$

\section{GPU-Oriented Random Rotation}

During a GPU timing attack, the only information available to the attacker is the execution time and kernel inputs. The attacker attempts to correlate the execution time with predicted number of transactions under each key guess in view of the coalecsing width. Another way to decorrelate these two parameters is to change the access pattern of the threads to memory, which results in a nondeterministic number of transactions. In order to change the access pattern, we should study the GPU's memory model. Based on how we map data in to the GPU memory, we can introduce nondeterminism to the access pattern.

In an AES encryption/decryption baseline attack, 5 T-table are copied from the CPU memory to the GPU memory. As

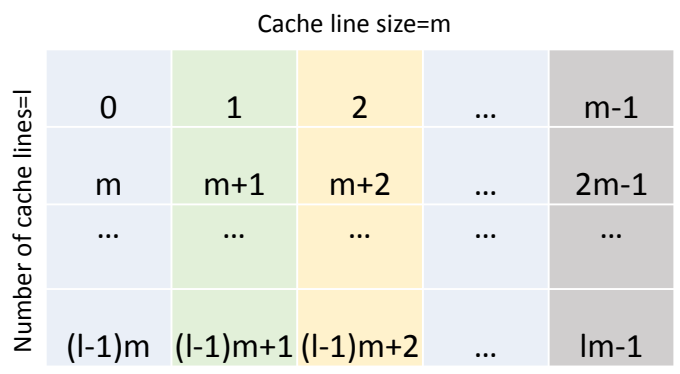

Fig. 6. General structure of a lookup table with size $n=m l$ in cache, where the cache line can hold $m$ elements.

the attack targets the last round of the encryption by tracking accesses to $T_{4}$, we will focus on mapping of this table to GPU memory. While we focus on accesses to $T_{4}$ due to their observability, we can apply rotation to any look-up table. A look-up table is a one-dimensional array of size $n$. If we assume the whole array can fit in the L1 cache, and each cache line can store $m$, then the table would occupy $l$ lines, where $l=\left[\frac{n}{m}\right]+1$. Figure 6 shows the general structure of this look-up table in cache. Whenever threads within a warp make a request to memory, the coalescing unit combines the requests within a cache line (without randomizing the width of coalescing unit) and will access the whole cache line. Therefore, the number of accessed cache lines depends on the row-wise location of the request. To introduce randomness to the number of transactions in this structure, we can dynamically change the mapping of an address to the cache.

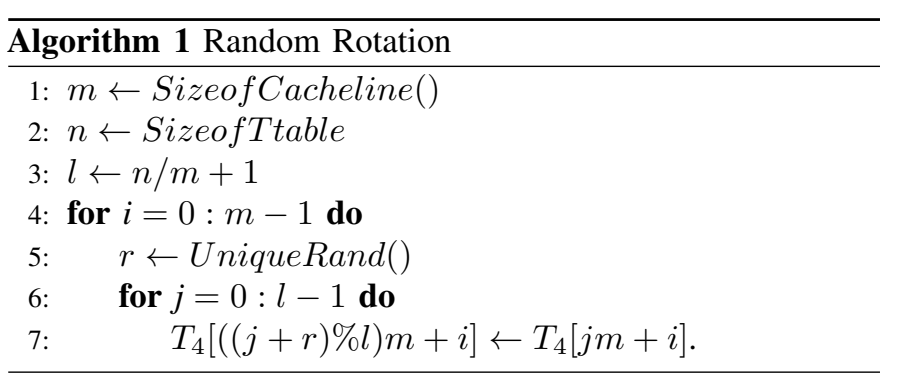

An example of mapping the look-up table is when we want to map element $(i, j)$ to $\left(i+i_{0}, j+j_{0}\right)$, which is in the range of $[m, l]$. But as discussed, the coalescing unit accesses the whole row and produces a deterministic memory behavior. But if we use a column-wise random rotation, we can introduce noise into the timing channel. Algorithm 1 provides pseudocode for rotation algorithm. Using this scheme, each column will circulate based on a unique randomly chosen number between $[0, l-1]$, providing the highest level of diffusion across the cache as possible.

The rotation can be performed on either the CPU or the GPU side. The main drawback of performing this transformation on the CPU side is that the rotation would be performed only one time, since it is too expense to repeat the memory transfer from the CPU to the GPU. When performed on the GPU side, this not only eliminates data transfer overhead, but also our 
rotation can be done in parallel on the many cores of the GPU. As a result, the performance overhead is much smaller, thus we use a GPU implementation, and can frequenty run a table rotation. The question of how often rotation is necessary is discussed next.

The last round of AES is the target for our attack. This round involves a number of look-up table accesses to table $T_{4}$. The size of the table is $1 \mathrm{~K}$ entries, occupying 16 64B cache lines. Suppose that we rotate columns in the T-table every 100,000 kernel executions. So every 100,000 iterations, the algorithm 1 is executed. Each thread is responsible for rotating a column of $4 \mathrm{~B}$ elements, 16 threads in total. Then the AES encryption will be started. Then, the probability of having the same number of transactions is significantly decreased, which reduces the correlation between execution time and number of transactions significantly.

\section{Countermeasure Analysis}

One approach to defend against a timing attack would result in an increase in the number of timing samples required to launch a successful attack. As we discussed earlier, the attacker uses the correlation between the execution time and number of transactions to find the key, so-called distinguisher. The attacker must be able to do this using a reasonable number of samples. Our series of countermeasures increase the number of required samples while keeping and in some cases even improving performance. Here, we first tried to find the number of samples for a successful attack using hardware and software countermeasures separately. Then we used all countermeasures together and tried to attack. As the number of samples increase significantly in this case, a detectable correlation was not observed in a reasonable amount of time. So we calculated the number of samples for this case using statistical analyses.

The probability of launching an attack can be calculated as Equation 10 [33], where $S$ is the number of samples and $\rho_{\text {peak }}$ is the highest detectable correlation value. We have modified this equation by replacing $\rho=0$ with $\rho=\rho_{\text {ave }}$, because when using a smaller number of samples, this value is not negligible.

$$
\alpha=\Phi\left(\frac{\frac{1}{2} \ln \frac{1+\rho_{\text {peak }}}{1-\rho_{\text {peak }}}-\frac{1}{2} \ln \frac{1+\rho_{a v e}}{1-\rho_{a v e}}}{\sqrt{\frac{2}{S-3}}}\right)
$$

Our countermeasures reduce $\rho_{\text {peak }}$, then the attacker would need to collect more timing samples in order to detect $\rho_{\text {peak }}$ in the correlation values. As we discussed earlier, our possible hardware countermeasure options include randomizing the width of coalescing unit, utilizing our hierarchical MHSR design, both of which can reduce the SNR. Equation 12 shows the contribution of noise when computing the correlation value between the execution and the number of memory transactions.

$$
\begin{array}{r}
\rho_{\text {peak }}\left(t_{e}, n\right)=\frac{\mu_{t_{e} n}-\mu_{t_{e}} \mu_{n}}{\sigma_{t_{e}} \sigma_{n}}=\frac{\mu_{\left(\beta_{1} n+\beta_{0}\right) n+\epsilon n}-\mu_{\beta_{1} n+\beta_{0}} \mu_{n}-\mu_{\epsilon} \mu_{n}}{\sigma\left(\beta_{1} n+\beta_{0}\right)^{\sigma_{n}+\sigma_{\epsilon} \sigma_{n}}} \\
\rho_{\text {peak }}\left(t_{e}, n\right)=\frac{\rho_{\text {peak }}\left(\beta_{1} n+\beta_{0}\right)}{\sqrt{1+\frac{1}{S N R}}}
\end{array}
$$

The highest possible value for $\rho_{\text {peak }}$ is 1 . In this case, the variables would be strongly correlated and the measurements would need to be free of any noise. When launching a timing attack on an unprotected GPU, the highest $\rho_{\text {peak }}$ that we can obtain is 0.4031 , which is limited only by the inherent noise in the timing channel. The highest value for $\rho_{\text {peak }}$, when employing a single (but randomly selected) coalescing unit width for each kernel run with average value of 32, is 0.2994 , as shown in Figure 4. If we change the coalescing unit width dynamically during runtime, the max $\rho_{\text {peak }}$ value is reduced to 0.0547 , meaning that using different sizes for different cache lines introduces even more noise. Finally employing our hierarchical MSHR design, the SNR is reduced to 0.0447. But in this case, in order to fully utilize all SMs, we increased the number of threads to 32 threadsperwarp $\times 2$ warpsper $S M \times$ $15 S M s$.

For this series of countermeasures, it is important to choose the right average width based on the normal distribution of memory access requests in order to achieve a good balance between performance and security. Figure 7 shows the number of timing samples and performance of different average widths. Performance is defined as $\frac{1}{\text { executionTime }}$. As we can see, using a dynamic coalescing width with hierarchical MSHRs improves the resiliency of the design to attacks, while also boosting performance. With this design, the effort required by the attacker (measured in the number of timing samples collected) to launch a successful attack increases by $1433 \mathrm{X}$. On the other side, we can enjoy a $7 \%$ performance improvement. Considering the fact that deceasing the average width makes the design harder to attack, while losing performance, the designer can choose different widths based on the performance/security tradeoff. For a design to introduce negligible performance overhead, we should select an average width no narrower than 16B. Adopting a value of $16 \mathrm{~B}$ will increase the difficulty of launching a successful attack by $175 \mathrm{X}$, while a more aggressive design that increases the attacker effort by $1433 \mathrm{X}$ would suffer a $3 \%$ loss in performance.

While hardware countermeasures help to obscure the relationship between the kernel execution time and the number of transactions by lowering SNR, employing our software scheme of rotating T-table columns will also decrease the correlation since this also changes the number of transactions. Equation 14 computes the correlation after applying T-table rotation in the original hardware without applying hardware countermeasures. In this equation, $t$ is the measured time, $n$ is the number of transactions to original table, $o$ in the number of transactions after rotation and $p_{o=n}$ is the probability of $o=n$. In this equation, we assume that the time is not related to $o$ whenever the number of transactions is not equal to $n$.

$$
\begin{array}{r}
\rho_{\text {peak }}(t, o)=\frac{p_{o=n}\left(\mu_{t n}-\mu_{t} \mu_{n}\right)+\left(1-p_{o=n}\right)\left(\mu_{t} \mu_{o}-\mu_{t} \mu_{o}\right)}{\sigma_{t} \sigma_{o}} \\
\rho_{\text {peak }}(t, o)=\rho_{\text {peak }}(t, n) p_{o=n} \frac{\sigma_{n}}{\sigma_{o}}
\end{array}
$$

The values for $p_{o=n}$ and $\sigma_{o}$ depend on the frequency of rotations. Using every 1000 encryptions as the frequency for 

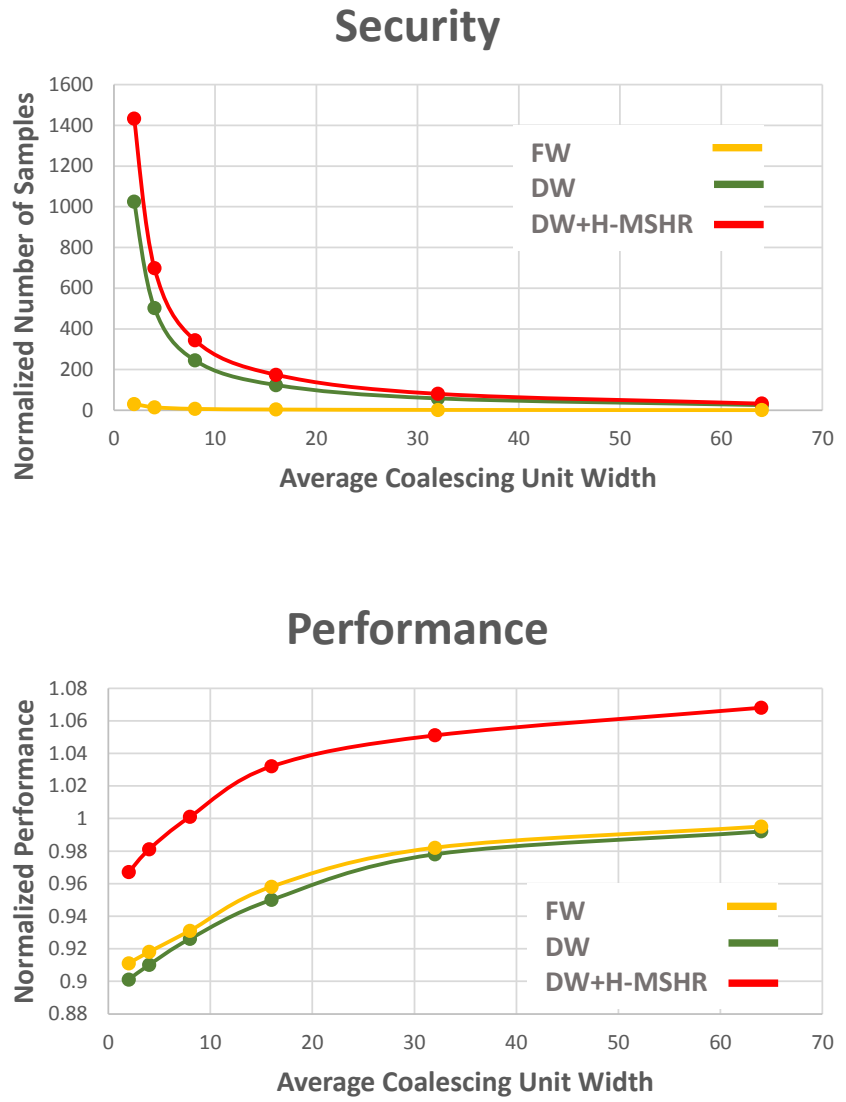

Fig. 7. Security and performance for hardware countermeasures.FX:Fixed Width, DW:Dynamic Width, H-MSHRs: Hierarchical MSHRs

rotation, $\rho_{\text {peak }}(t, o)$ can be decreased to $0.0858 \times \rho_{\text {peak }}(t, n)$, without applying any hardware countermeasures. Figure 8 shows the resulting security and performance values based on the rotation frequency. This figure shows that rotating $\mathrm{T}$ table columns every $1 \mathrm{M}$ to 1000 samples, we do not see any detectable performance loss, but increases the number of samples by $68 \mathrm{X}$. Decreasing the time between rotations to less than 1000 encryptions would result in a slight performance loss. Using the maximum frequency, the T-table will be rotated after every run of the encryption algorithm, resulting in a $2 \%$ performance loss, while increasing the effort for a successful timing attack by $178 \mathrm{X}$.

Figure 9 shows the correlation values when attacking key byte 5 , As we can see, the number of samples required for the original attack with $\alpha=0.9$ is around $5 \times 10^{5}$. When we apply the hardware (average width=32B )and software (rotation frequency $=1000$ ) countermeasures individually, the number of traces needed grows to $4 \times 10^{7}$ and $3.5 \times 10^{7}$, respectively, increasing the effort by two orders of magnitude.

Our hardware and software methods reduce the correlation to $0.1109 \times p_{\text {peak }}$ and $0.0858 \times p_{\text {peak }}$, respectively. When we apply both methods, the correlation will be at least $0.1109 \times$ $0.0858 \times p_{\text {peak }}$. By reducing the width of the coalescing unit, the number of cache lines accessed will increase, which will decrease $p_{o=n}$. For example, with a 64B L1 cache line, 32

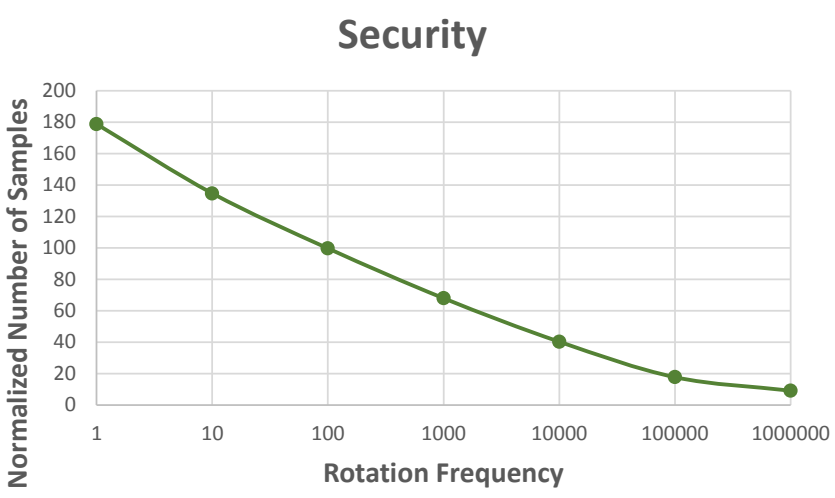

Performance

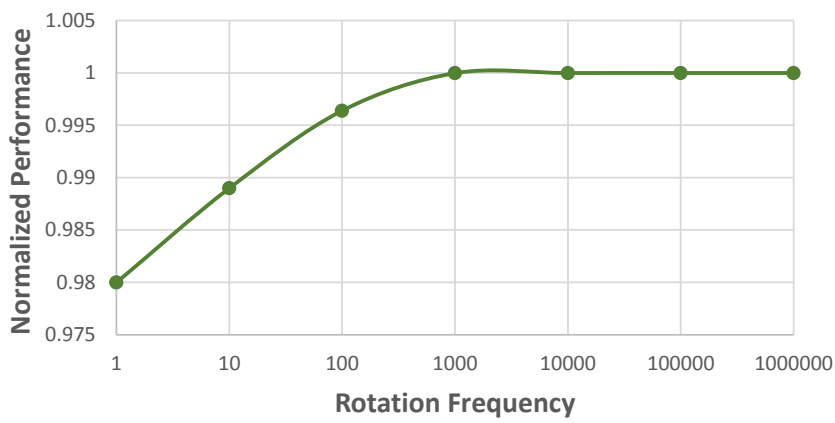

Fig. 8. Security and performance for software countermeasures.
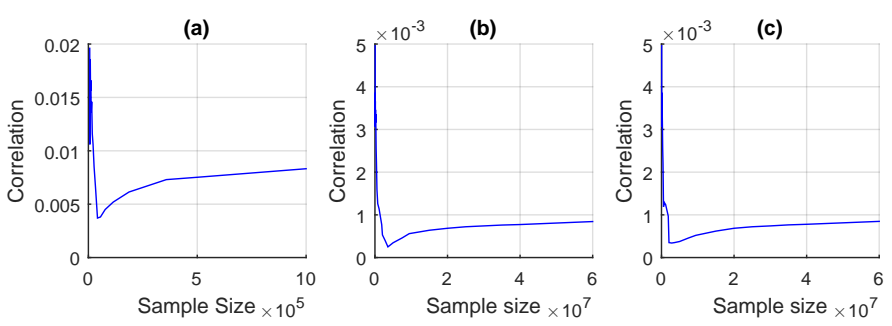

Fig. 9. $\rho_{\text {peak }}$ of $k_{5}$ vs. the number of samples a)the baseline attack, b)the attack only with hardware countermeasures, c)the attack only with software countermeasures

requests will access 2 to 16 cache lines. To change each number from this range, we have 14 other possible values. But having 32B cache lines results in accessing 4 to 64 cache lines. So the possibility of hiding the original number of cache lines is higher due to using a wider range. Therefore, hardware obfuscation will increase the level of obfuscation performed in software. If the number of required samples with hardware and software countermeasures individually are $g_{h} X$ and $g_{s} X$, respectively (as compared to the traces required for the basic attack), the number of required traces needed to launch a successful attack using a combination of both countermeasures will be increased by at least $g_{h} * g_{s}$ times.

\section{CONCLUSION}

GPUs are vulnerable to timing side-channel attacks. The memory system of these devices can leak timing information. 
If the attacker can time the encyrption, and with general knowledge of an encryption algorithm's memory access pattern, she can recover all bytes of the key.

In this paper we explore multiple hardware-based and software-based strategies to obfuscate the timing leakage of the memory system on a GPU. By randomizing the width of the coalescing unit dynamically, we are able to introduce significant noise into the timing channel. By introducing a second-level unified MSHRs across all SMs, we can further obfuscate the timing channel, while improving encyption performance. We also propose software-based methods the obfuscate T-table accesses and the mapping of entries to cache lines. Applying all of these countermeasures together to thwart an attack, an attacker will still be able to recover the encryption key, but the number of traces required for a successful attack has increased by $1400 * 178 \mathrm{X}$ over the baseline, while slightly improving AES performance.

\section{REFERENCES}

[1] M. Aaftab, "The opencl specification," in Hot Chips 21 Symposium (HCS), 2009 IEEE, pp. 1-314, IEEE, 2009.

[2] D. R. Kaeli, P. Mistry, D. Schaa, and D. P. Zhang, Heterogeneous Computing with OpenCL 2.0. San Francisco, CA, USA: Morgan Kaufmann Publishers Inc., 1st ed., 2015.

[3] J. Nickolls, I. Buck, M. Garland, and K. Skadron, "Scalable parallel programming with cuda," in ACM SIGGRAPH 2008 classes, p. 16, ACM, 2008.

[4] N. Corporation, "Gpu-accelerated applications," 2018.

[5] N. Corporation, "Nvidia cuda toolkit v10.0 documentation," 2018.

[6] S. A. Manavski et al., "Cuda compatible gpu as an efficient hardware accelerator for aes cryptography," Signal Processing and Communications, vol. 2007, 2007.

[7] K. Iwai, T. Kurokawa, and N. Nisikawa, "Aes encryption implementation on cuda gpu and its analysis," in 2010 First International Conference on Networking and Computing, pp. 209-214, IEEE, 2010.

[8] A. D. Biagio, A. Barenghi, G. Agosta, and G. Pelosi, "Design of a parallel aes for graphics hardware using the cuda framework," in Proceedings of the 2009 IEEE International Symposium on Parallel\&Distributed Processing, pp. 1-8, IEEE Computer Society, 2009.

[9] D. Le, J. Chang, X. Gou, A. Zhang, and C. Lu, "Parallel aes algorithm for fast data encryption on gpu," in Computer Engineering and Technology (ICCET), 2010 2nd International Conference on, vol. 6, pp. V6-1, IEEE 2010.

[10] A. E. Cohen and K. K. Parhi, "Gpu accelerated elliptic curve cryptography in gf $(2 \mathrm{~m})$, , in 2010 53rd IEEE International Midwest Symposium on Circuits and Systems, pp. 57-60, IEEE, 2010.

[11] R. Szerwinski and T. Güneysu, "Exploiting the power of gpus for asymmetric cryptography," in International Workshop on Cryptographic hardware and embedded systems, pp. 79-99, Springer, 2008.

[12] Q. Li, C. Zhong, K. Zhao, X. Mei, and X. Chu, "Implementation and Analysis of AES Encryption on GPU," in IEEE 14th International Conference on High Performance Computing and Communication, pp. 843-848, June 2012.

[13] G. Schönberger and J. Fuß, "GPU-assisted AES Encryption Using GCM," in Proceedings of the 12th IFIP TC 6/TC 11 International Conference on Communications and Multimedia Security, pp. 178-185, Springer-Verlag, 2011.

[14] M. Patterson, "Vulnerability analysis of gpu computing," 2013.
[15] E. Karimi, Z. H. Jiang, Y. Fei, and D. Kaeli, "A timing side-shannel atack on a mobile gpu.," in ICCD, vol. 2018, 2018.

[16] Z. H. Jiang, Y. Fei, and D. Kaeli, "A complete key recovery timing attack on a gpu," in High Performance Computer Architecture (HPCA), 2016 IEEE International Symposium on, pp. 394-405, IEEE, 2016.

[17] Z. H. Jiang, Y. Fei, and D. Kaeli, "A novel side-channel timing attack on gpus," in Proceedings of the on Great Lakes Symposium on VLSI 2017, pp. 167-172, ACM, 2017.

[18] K. Akdemir, M. Dixon, W. Feghali, P. Fay, V. Gopal, J. Guilford, E. Ozturk, G. Wolrich, and R. Zohar, "Breakthrough aes performance with intel aes new instructions," White paper, June, p. 11, 2010.

[19] G. Kadam, D. Zhang, and A. Jog, "Rcoal: mitigating gpu timing attack via subwarp-based randomized coalescing techniques," in High Performance Computer Architecture (HPCA), 2018 IEEE International Symposium on, pp. 156-167, IEEE, 2018.

[20] A. Bakhoda, G. L. Yuan, W. W. L. Fung, H. Wong, and T. M. Aamodt, "Analyzing cuda workloads using a detailed gpu simulator," in IEEE International Symposium on Performance Analysis of Systems and Software, pp. 163-174, April 2009.

[21] L. Wang, X. Zhao, D. Kaeli, Z. Wang, and L. Eeckhout, "Intra-cluster coalescing to reduce gpu noc pressure," in 2018 IEEE International Parallel and Distributed Processing Symposium (IPDPS), pp. 990-999, IEEE, 2018.

[22] J. Tuck, L. Ceze, and J. Torrellas, "Scalable cache miss handling for high memory-level parallelism," in Proceedings of the 39th Annual IEEE/ACM International Symposium on Microarchitecture, pp. 409-422, IEEE Computer Society, 2006.

[23] S. Keshavarz and D. Holcomb, "Privacy leakages in approximate adders," in 2017 IEEE International Symposium on Circuits and Systems (ISCAS), pp. 1-4, IEEE, 2017.

[24] S. Keshavarz, F. Schellenberg, B. Richte, C. Paar, and D. Holcomb, "Satbased reverse engineering of gate-level schematics using fault injection and probing," in 2018 IEEE International Symposium on Hardware Oriented Security and Trust (HOST), pp. 215-220, IEEE, 2018.

[25] S. Keshavarz, C. Paar, and D. Holcomb, "Design automation for obfuscated circuits with multiple viable functions," in Design, Automation \& Test in Europe Conference \& Exhibition (DATE), 2017, pp. 886-889, IEEE, 2017

[26] M. A. Vosoughi and S. Köse, "Combined distinguishers to enhance the accuracy and success of side channel analysis," in 2019 IEEE International Symposium on Circuits and Systems (ISCAS), pp. 1-5, IEEE, 2019.

[27] M. A. Vosoughi, L. Wang, and S. Köse, "Bus-invert coding as a lowpower countermeasure against correlation power analysis attack," in 2019 ACM/IEEE International Workshop on System Level Interconnect Prediction (SLIP), pp. 1-5, IEEE, 2019.

[28] A. Vosoughi and S. Köse, "Leveraging on-chip voltage regulators against fault injection attacks," in Proceedings of the 2019 on Great Lakes Symposium on VLSI, pp. 15-20, ACM, 2019.

[29] D. J. Bernstein, "Cache-timing attacks on aes," 2005.

[30] E. Tromer, D. A. Osvik, and A. Shamir, "Efficient cache attacks on aes, and countermeasures," Journal of Cryptology, vol. 23, no. 1, pp. 37-71, 2010.

[31] Z. Wang and R. B. Lee, "New cache designs for thwarting software cachebased side channel attacks," ACM SIGARCH Computer Architecture News, vol. 35, no. 2, pp. 494-505, 2007.

[32] E. Brickell, G. Graunke, M. Neve, and J.-P. Seifert, "Software mitigations to hedge aes against cache-based software side channel vulnerabilities.," IACR Cryptology ePrint Archive, vol. 2006, p. 52, 2006.

[33] S. Mangard, "Hardware countermeasures against dpa-a statistical analysis of their effectiveness," in Cryptographers Track at the RSA Conference, pp. 222-235, Springer, 2004. 